\title{
Tratamiento integral de la obesidad infantil: Efecto de una intervención psicológica
}

\author{
M. ISABEL SALAS A. ${ }^{a}$, VIVIEN GATTAS Z. ${ }^{b}$, \\ XIMENA CEBALLOS S. ${ }^{\mathrm{b}}$, RAQUEL BURROWS A.
}

\section{Effects of psychological support as an adjunct to a weight reducing program among obese children}

Background: Disrupted eating behaviors and psychological issues can have a role in the development of childhood obesity. Aim: To assess the effects of psychological support as an adjunct to the treatment of obesity among children. Material and Methods: Retrospective analysis of a group of 152 obese children aged 3 to 16 years, who received medical, nutritional and physical training treatment. Seventy three (Group I) were invited to participate in a protocol of psychological support and 79 formed the control group (Group 2). Body mass index, body fat mass, waist circumference, insulin sensitivity, lipid profile, eating and physical activity habits were evaluated. Family structure, history of food rejection, psychological, physical and social development, and adherence to the program were also analyzed. To analyze responses to treatment, Group I was further divided into those who attended the psychology sections (intervened group) and those who did not attend (non intervened group). Results: At baseline, there were no differences in anthropometric, metabolic, familial and psychological profile among Groups I and 2. Family structure, eating behaviors and physical and social development were normal. Those associated with lack of control appeared as negative characteristics. There was a significant improvement in eating habits and physical activity among intervened children of Group 1 and Group 2. No significant change in weight was recorded in all three groups. Compliance with the program was significantly better among intervened children in Group 1 (62.1\% at sixth months), compared with non intervened children of Group 1 (15.9\%) and Group 2 (30.3\%). Conclusions: Obese children who attended psychological support therapy as an adjunct to a weight reducing program, had a better compliance with treatment but did not achieve a higher weight loss.

(Rev Med Chile 2010; 138: 1217-1225).

Key words: Childhood; Obesity; Psychological techniques.

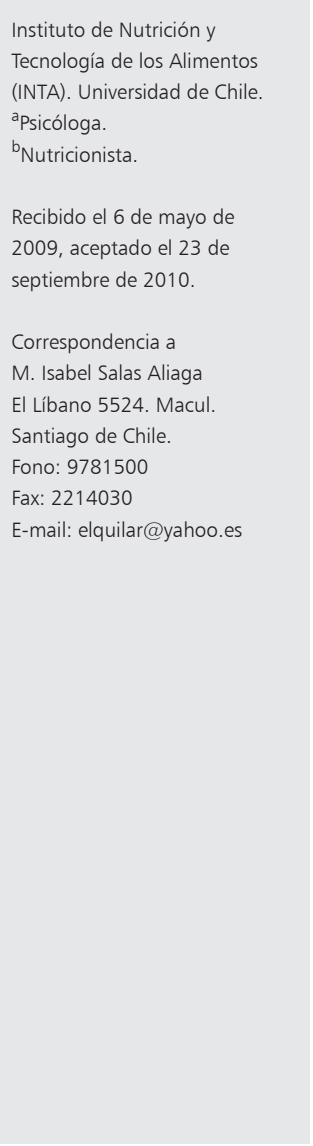

E 1 modelo de desarrollo económico vigente, ha cambiado drásticamente nuestros estilos de vida, entre estos, el patrón de alimentación y de actividad física, con el consecuente aumento de la obesidad y de sus co-morbilidades ${ }^{1}$. La obesidad infantil se cuadruplicó entre los años 1985 y $2000^{2-5}$. La ingesta aumentada de alimentos hipercalóricos, la jornada escolar completa, el marketing televisivo y la inactividad física, estarían contribuyendo a la mantención del sobrepeso ${ }^{6-9}$.

Se reconoce a la obesidad infantil como una enfermedad crónica, porque se perpetúa en el tiempo y se asocia a un síndrome metabólico (SM) con un mayor riesgo de Diabetes 2 y cardiopatías coronarias $^{10,11}$. Estudios nacionales señalan que $30 \%$ de los niños que consultan por obesidad 
tienen $\mathrm{SM}^{12}$. El tratamiento temprano de la obesidad sería la mejor estrategia para su prevención, sin embargo, constituye un difícil desafío que requiere de cambios a nivel individual, familiar, educacional y social ${ }^{13}$.

Los resultados de las intervenciones no son alentadores, aunque coinciden en que el tratamiento integral, con énfasis en el cambio en los hábitos de alimentación y de actividad física tendría los mejores resultados ${ }^{14,15}$. En este contexto, el abordaje de la dimensión psicológica constituye una tarea principal ${ }^{16}$. Los estudios que abordan la relación entre variables de orden psicológico y obesidad, establecen fundamentalmente una relación de causalidad entre determinadas características psicológicas y la obesidad, señalando ciertas generalidades respecto del manejo de variables de orden psicológico, sin dar cuenta de una relación de impacto entre intervención y cambio. Entre estos estudios podemos distinguir tres grupos: 1) aquellos que relacionan la conducta de sobreingesta con variables psicológicas asociadas a la personalidad; 2) aquellos que la relacionan con determinados mecanismos de control (procesos regulatorios) y 3) aquellos que dicen relación con variables de contexto relacional (estilos de vida $)^{17}$. Entre estos últimos, los más recientes describen patrones de funcionamiento familiar caracterizados por dificultades de control, manejo emocional y promoción de la autonomía entre sus miembros ${ }^{18,19}$.

Sobre la base de estos antecedentes, establecimos una intervención que abordara la dimensión psicológica. El objetivo de este estudio fue comparar la respuesta a un tratamiento clínico de la obesidad de niños que participaron de un protocolo con apoyo psicológico versus la de niños que no participaron de dicho protocolo.

\section{Material y Método}

Estudio descriptivo y analítico que corresponde a un análisis posterior de una muestra de niños entre 3 y 16 años que consultaron voluntariamente al Programa Clínico de Obesidad Infantil del INTA entre los años 2000 y 2001. Por ese motivo, no se solicitó consentimiento a los participantes y no fue sometido al comité de ética del INTA. Este programa contempla un protocolo médico, nutricional, de actividad física y psicológico teniendo como objetivos: 1) modificar conductas de ingesta y actividad física, promoviendo hábitos saludables en el paciente y su familia; 2) mejorar la condición física; 3 ) disminuir los factores de riesgo cardiovascular asociados y 4) apoyar psicológicamente al niño y su familia, fortaleciendo procesos autoregulatorios en los niños y mejorando estrategias de control familiar. El tiempo total del programa es de 12 meses y contempla control con médico y nutricionista (8 consultas) con preparador físico (3 consultas) y con psicólogo (1 consulta y 12 sesiones grupales). En cada niño se evalúa al ingreso y trimestralmente el IMC, midiendo el peso y la talla con una balanza electrónica de precisión (SECA) con cartabón incluido, el porcentaje de grasa corporal total (\% GCT) por sumatoria de 4 pliegues cutáneos ${ }^{20}$, el perímetro de la cintura (PC) con huincha de fijación automática (SECA), la presión arterial diastólica (PAD) y sistólica (PAS) según metodología estandarizada por el "Second task Force" 21 , la calidad de los hábitos de ingesta (HI) y actividad física (HAF) por encuesta ad hoc validada por los autores, que los pondera con un puntaje entre 0 y 10 puntos, señalando en sentido creciente una mejor calidad ${ }^{8,22}$. En todos los niños se evalúa al ingreso y en ayunas la glicemia por método enzimático colorimétrico GOD-PAP (Química Clínica Aplicada S.A.), la insulinemia (RIA Diagnostic Products Corporation), el perfil de colesterol (metodología analítica seca Vitros, Johnson \& Johnson, Clinical diagnostics Inc) y la sensibilidad insulínica a través del HOMA-IR ${ }^{23}$.

El protocolo de intervención psicosocial establece dos unidades de intervención: niño y familia. Tiene como objetivos generales: a) abordar con los niños aspectos psicológicos involucrados en el sobrepeso y b) integrar la participación del niño y su familia en el programa, a través de un trabajo complementario con sus padres. Los objetivos específicos en los niños son: a) identificar y distinguir sensaciones, necesidades, emociones e ideas; b) modular expresión conductual y emocional; c) entrenar habilidades sociales; d) fortalecer el desarrollo del auto-concepto y autoestima, y e) fortalecer los procesos de autonomía e individuación. En los padres: a) fortalecer su rol parental y b) identificar "facilitadores" y "obstaculizadores" de los procesos de autonomía e individuación. La intervención consta de 12 sesiones (9 con los niños y 3 con sus padres) y se realiza conformando grupos cerrados, mixtos, de acuerdo a edad, con un máximo de 12 participantes. 
De los 152 niños ingresados entre los años 2000-2001, 73 fueron convocados a estos talleres de intervención psicosocial (Grupo 1) y los 79 restantes, conformaron el grupo control (Grupo 2). No fueron convocados niños pequeños (menores de 6 años), o de edades de baja frecuencia, que no permitían la conformación de un grupo, así como quienes residían fuera de la ciudad.

\section{Análisis estadístico}

Se realizaron pruebas de bondad de ajuste y homogeneidad de varianza. Los resultados se expresaron como mediana, p25, p75, promedio \pm DE. Se utilizó Chi cuadrado para comprar grupos (Willcoxon, Fisher), Odds Ratio para calcular riesgo y análisis de varianza de clasificación simple y la prueba de comparación múltiple de Friedman para comparar los niños que recibieron la intervención psicosocial vs los que no la recibieron. Se estableció en un $\mathrm{p}<0,05$ el punto de corte para la significancia estadística.

\section{Resultados}

No hubo diferencias significativas entre el Grupo 1 y 2, en las características generales (Tabla 1), ni en el perfil metabólico y cardiovascular (Tabla 2) al ingreso al programa. Tampoco hubo diferencias entre ambos grupos en la estructura familiar (Figuras $1 \mathrm{~A}$ y B). Hubo una importante proporción de familias de tipo nuclear $(69,8$ y $73,4 \%$ respectivamente) y sistema biparental $(76,7$ y $78,5 \%$ respectivamente). Con respecto a antecedentes del desarrollo de los niños (Figura 2A), tampoco hubo diferencias entre ambos grupos y la mayoría son percibidos por sus padres como normales, tanto en el área física $(72,9$ y $72,4 \%$ respectivamente), psicológica $(75,7$ y $63,2 \%$ respectivamente) como social ( 77,1 y $73,7 \%$ respectivamente). De la
Tabla 1. Características generales de la muestra al ingreso al programa

\begin{tabular}{|lcccc|}
\hline & Grupo 1 (73) & Grupo 2 (79) & Pc \\
\hline Edad (años) & $10,3(9,1-12,3)^{\mathrm{b}}$ & $10,6(6,6-12,3)$ & n.s \\
\hline Test de ingesta (ptje) & $4,0(3,0-6,0)$ & $4,5(3,0-6,0)$ & n.s \\
\hline Test de AF (ptje) & $2,0(1,0-3,0)$ & $2,0(2,0-3,0)$ & n.s \\
IMC (ptje z) & $4,0(2,9-5,0)$ & $4,3(3,1-5,5)$ & n.s \\
GCT (\%) & $34,8(31,4-38,1)$ & $33,0(28,2-38,3)$ & n.s \\
Cintura (cm) & $89,9(83,0-96,0)$ & $88,5(75,5-100)$ & n.s \\
\hline
\end{tabular}

anúmero de casos; ' Mediana (P25-P75); 'significancia según Kruskal-Wallis.

Tabla 2. Perfil metabólico y cardiovascular de la muestra al ingreso al programa

\begin{tabular}{|lccc|}
\hline & Grupo 1 (73) & Grupo 2 (79) & Pc \\
PAS & $110(100-120)$ & $105(100-120)$ & n.s \\
PAD & $70(60-70)$ & $70(60-75)$ & n.s \\
Triglicéridos (mg/dl) & $99,5(71-155)$ & $85,5(71-129)$ & n.s \\
HDL- Col (mg/dl) & $44(38-50)$ & $45,5(38-52)$ & n.s \\
Glicemia (mg/dl) & $87(82-91)$ & $86(80-92)$ & n.s \\
Insulina (uUl/dl) & $14,1(11-20,7)$ & $11,3(6,4-22,9)$ & n.s \\
HOMA & $3,2(2,3-4,5)$ & $2,3(1,4-4,2)$ & n.s \\
\hline
\end{tabular}

aNúmero de casos; cSignificancia según Kruskal-Wallis.

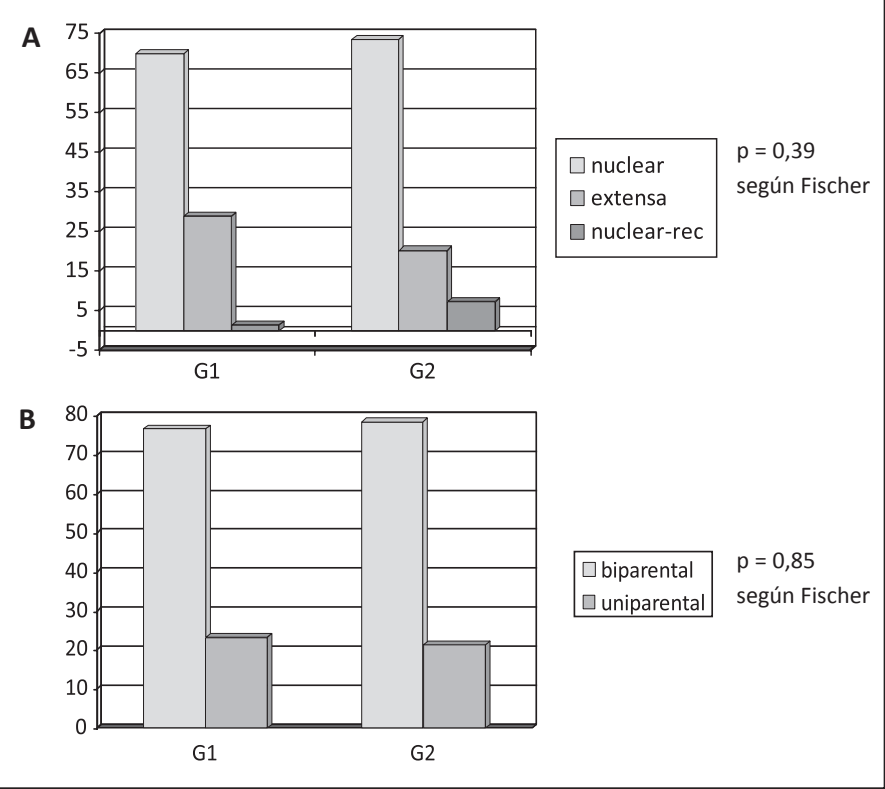

Figura 1. Estructura familiar: tipo de familia (A) y presencia del padre (B) en los Grupos 1 y 2. 
A

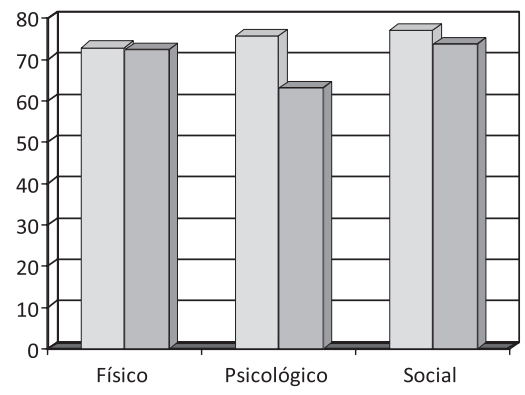

$\mathrm{p}=\mathrm{n} \cdot \mathrm{s}$

según Fischer

\section{$\square$ Grupo $1 \quad \square$ Grupo 2}

B

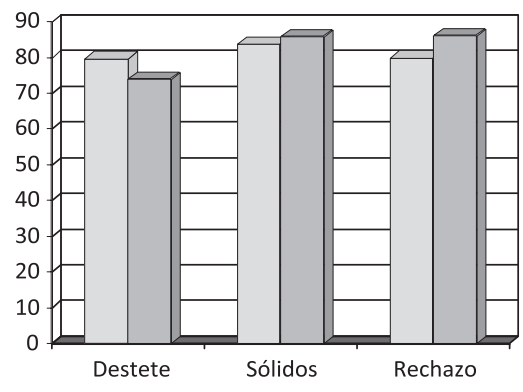

$\mathrm{p}=$ n.s
según Fischer misma forma, no hubo diferencias entre el Grupo 1 y 2 en las características de la alimentación temprana (Figura 2B) y la gran mayoría no presentó dificultad en el destete $(79,7$ y $74,0 \%)$, en la introducción de sólidos $(83,8$ y $85,7 \%)$ ni rechazo alimentario $(81,2$ y $86,1 \%)$. En relación con las principales características conductuales de los niños, tampoco hubo diferencias significativas entre los grupos ( $\mathrm{Fi}$ gura 3). Los padres los describieron principalmente con atributos positivos (cariñosos, inteligentes, alegres, bondadosos) y en menor proporción, refieren características de desregulación (enojón, rabioso, lábil).

De los 73 niños del Grupo 1, sólo $29(39,7 \%)$ asistieron a lo menos a 5 de las 9 sesiones, y constituyen para el análisis que viene a continuación, el Grupo 1 intervenido (G1I). Los 44 restantes no asistieron a los talleres $(60,3 \%)$ o asistieron a un número de sesiones menor al corte de intervención establecido, y constituyen para
Figura 2. Antecedentes de normalidad del desarrollo (A) y de la alimentación temprana (B) en los Grupos 1 y 2.

Grupo 1

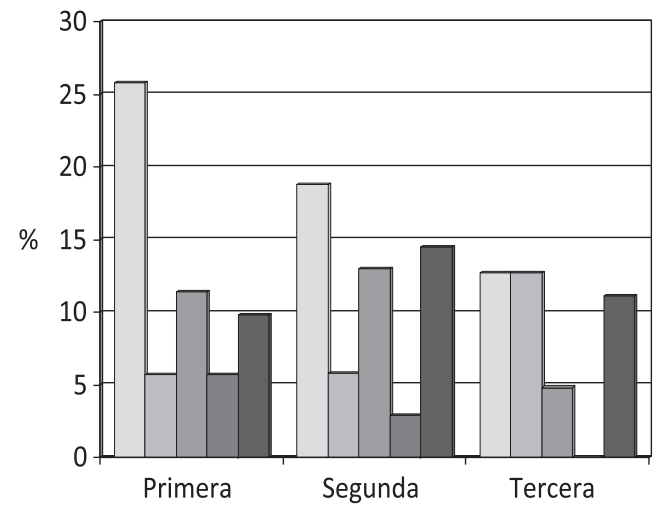

Grupo 2

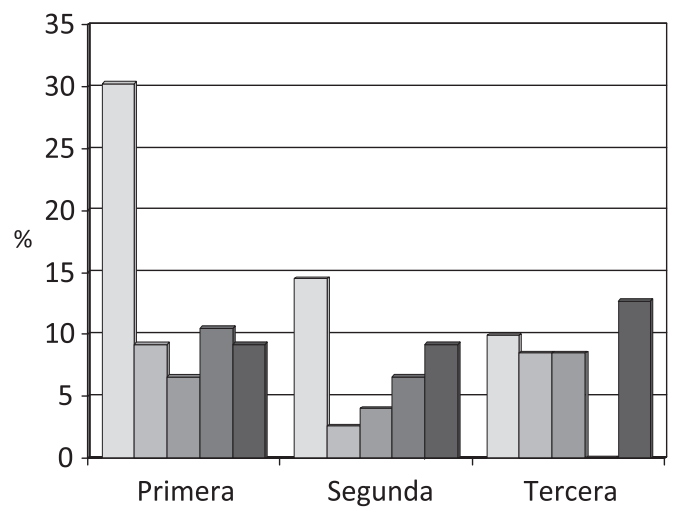

$\square$ Cariñoso $\square$ Bondadoso $\quad \square$ Inteligente $\quad \square$ Alegre $\quad \square$ Desregulado

Figura 3. Principales características conductuales en niños obesos, según percepción de los padres. 
Intervención psicológica en niños obesos - M. I. Salas et al

Tabla 3. Características generales del Grupo 1 al ingreso al programa según asistencia al taller

\begin{tabular}{|llll|}
\hline & \multicolumn{1}{c}{$\begin{array}{c}\text { G1 intervenido } \\
(\mathbf{2 9})^{\mathbf{a}}\end{array}$} & $\begin{array}{c}\text { Grupo } \mathbf{1} \text { no inter- } \\
\text { venido NI (44) }\end{array}$ & Pc \\
\hline Edad (años) & $9,9(9,4-11,5)^{\mathrm{b}}$ & $10,5(9-13)$ & n.s \\
$\begin{array}{l}\text { Tiempo de obesidad } \\
\text { (años) }\end{array}$ & $5,0(4-9)$ & $5,0(3-8)$ & n.s \\
\hline Test de ingesta (ptje) & $4,0(3,5-6,0)$ & $4,0(3,5-6,0)$ & n.s \\
Test de AF (ptje) & $2,0(1,0-3,0)$ & $2,0(1,0-3,0)$ & n.s \\
IMC (ptje z) & $3,6(2,8-4,7)$ & $4,1(3,1-5,2)$ & n.s \\
GCT (\%) & $33,0(29,6-35,2)$ & $36,8(33-41)$ & n.s \\
\hline Cintura (cm) & $85,5(80,0-92,3)$ & $89,0(84-99)$ & n.s \\
IMC Papá & $29,4(26,5-31,2)$ & $26,8(26-30,2)$ & n.s \\
IMC Mamá & $24,8(23,3-29,5)$ & $25,8(23-28,6)$ & n.s \\
\hline
\end{tabular}

aNúmero de casos; 'bediana (P25-P75); 'Significancia según Willcoxon (Mann-Whitney)

Tabla 4. Perfil metabólico y cardiovascular del Grupo 1 al ingreso al programa según asistencia a talleres

\begin{tabular}{|lccc|}
\hline & $\begin{array}{c}\text { Grupo 1 } \\
\text { intervenido (29) }\end{array}$ & $\begin{array}{c}\text { Grupo 1 no } \\
\text { intervenido (43) }\end{array}$ & Pc \\
\hline PAS & $110(100-110)$ & $110(103-120)$ & n.s \\
PAD & $70(60-75)$ & $70(60-70)$ & n.s \\
\hline Triglicéridos (mg/dl) & $97,0(63-144)$ & $105,5(85-167)$ & n.s \\
HDL- Col (mg/dl) & $46(41-52)$ & $43(37-47,4)$ & n.s \\
Glicemia (mg/dl) & $86(84-92)$ & $87(80-90)$ & n.s \\
Insulina (uUl/dl) & $12,4(9-18)$ & $16,1(12-22)$ & n.s \\
\hline HOMA & $2,7(2,0-4,1)$ & $3,4(2,5-4,6)$ & n.s \\
\hline
\end{tabular}

${ }^{a}$ Número de casos; ' ${ }^{b}$ Mediana (P25-P75); 'Significancia según Willcoxon (Mann-Whitney). el análisis que viene a continuación el Grupo 1 no intervenido (G1NI). En el G1I, sólo 6,9\% (2) de los padres no asistió a las sesiones de los padres y $51,3 \%$ (15) asistió al menos a 2 de las 3 sesiones.

Con relación a las características generales (Tabla 3) y al perfil metabólico y cardiovascular (Tabla 4) del ingreso, no hubo diferencias significativas al comparar el G1I con el G 1NI.

La Tabla 5 muestra las variaciones trimestrales de los hábitos y de la antropometría durante los primeros 6 meses de tratamiento del G1I, del G1NI y del Grupo 2. En los 3 grupos se observa una tendencia a mejorar tantos los HI y HAF, como a disminuir el IMC, el PC y el \%GCT. Sin embargo, esta tendencia fue significativa sólo para los hábitos en el G1I y en el Grupo 2.

$\mathrm{Al}$ analizar la adherencia al Programa, hubo diferencias significativas entre los 3 grupos (Figura 4). A los 6 meses, $62,1 \%$ de los niños del Grupo 1I continuaban en el programa, vs $15,9 \%$ del Grupo 1 NI y 30,3\% del Grupo 2, con un OR de 3,9 al comparar Grupo 1I con el Grupo 1NI y de 2,0 al comparar grupo 1I con el Grupo 2. El 100\% de los niños del G1NI había desertado a los 9 meses, en tanto $24,1 \%$ de los niños del Grupo 1I completó 12 meses.

Tabla 5. Variaciones trimestrales de los hábitos y de parámetros antropométricos en los 3 grupos

\begin{tabular}{|lccccccccc|}
\hline Variable & \multicolumn{3}{c}{ G1 Intervenido (18) } & \multicolumn{2}{c}{ Grupo $\mathbf{1}$ no intervenido (7) } & \multicolumn{3}{c|}{ Grupo 2 (24) } \\
& Basal & $\mathbf{3}$ meses & $\mathbf{6}$ meses & Basal & $\mathbf{3}$ meses & $\mathbf{6}$ meses & Basal & $\mathbf{3}$ meses & $\mathbf{6}$ meses \\
\hline Ingesta (ptos) & $4,4 \pm 2,1^{* *}$ & $7,3 \pm 1,9$ & $7,5 \pm 1,6$ & $4,5 \pm 1,9$ & $6,3 \pm 2,9$ & $7,0 \pm 1,8$ & $4,6 \pm 1,7^{* *}$ & $6,6 \pm 2,1$ & $7,3 \pm 1,7$ \\
AF $^{1}$ (ptos) & $2,1 \pm 1,4^{*}$ & $3,3 \pm 1,5$ & $3,6 \pm 1,5$ & $1,4 \pm 0,5$ & $1,8 \pm 1,5$ & $2,6 \pm 0,9$ & $2,8 \pm 1,5^{*}$ & $3,6 \pm 1,4$ & $4,1 \pm 1,7$ \\
IMC (ptje z) & $4,2 \pm 1,8$ & $3,7 \pm 1,8$ & $3,3 \pm 1,5$ & $4,8 \pm 1,7$ & $4,7 \pm 2,1$ & $4,2 \pm 1,7$ & $4,7 \pm 2,3$ & $4,0 \pm 1,7$ & $3,6 \pm 2,1$ \\
Cintura (cms) & $89 \pm 12$ & $86 \pm 13$ & $86 \pm 12$ & $94 \pm 11$ & $97 \pm 16$ & $91 \pm 13$ & $89 \pm 15$ & $88 \pm 15$ & $85 \pm 14$ \\
GCT (\%) & $32 \pm 5$ & $31 \pm 6$ & $31 \pm 6$ & $38 \pm 6$ & $37 \pm 7$ & $35 \pm 6$ & $33 \pm 6$ & $32 \pm 7$ & $30 \pm 8$ \\
\hline
\end{tabular}

1 actividad física; Test de Friedman ${ }^{*} p<0,05 ;{ }^{*} p<0,001$. 

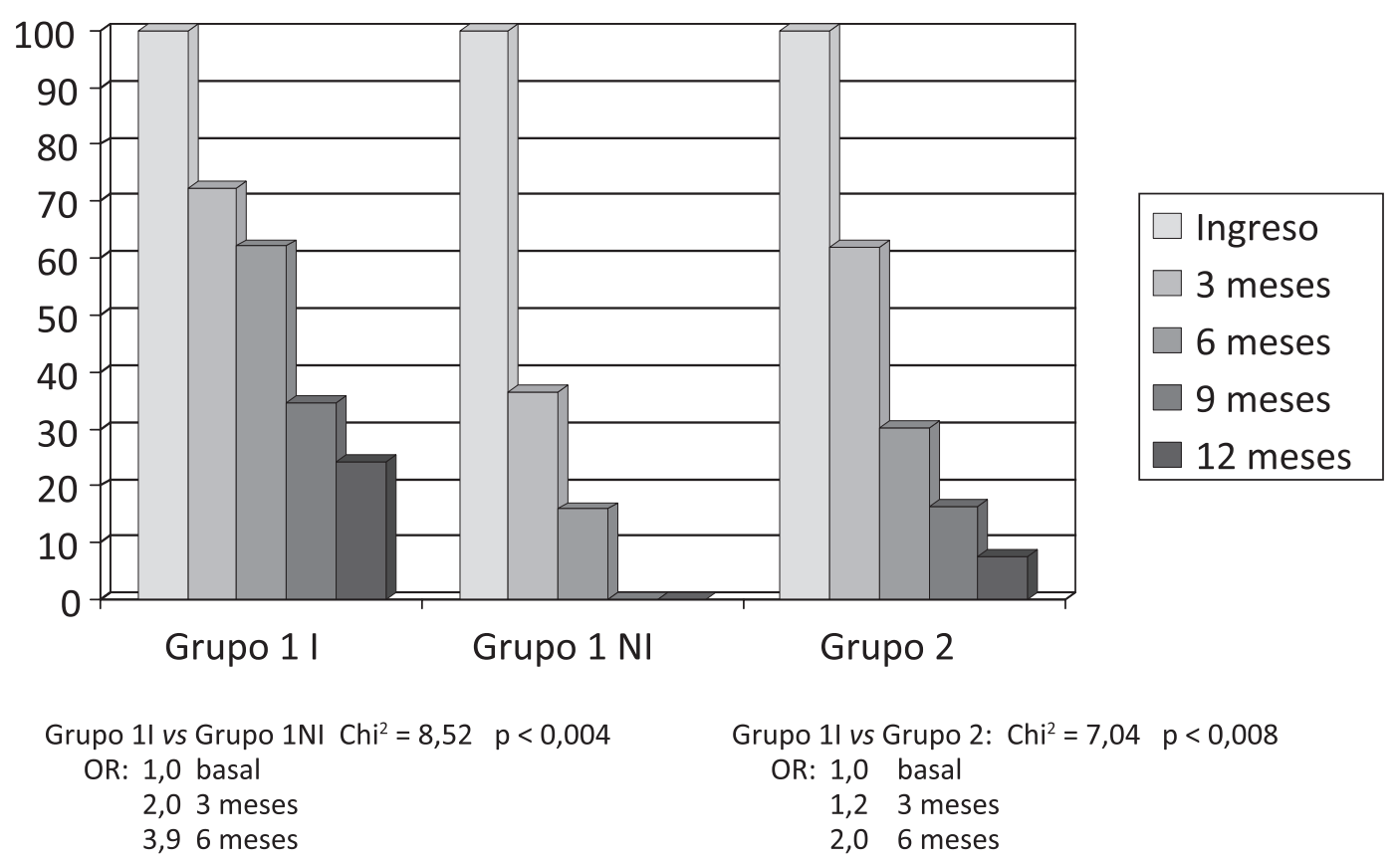

Grupo 1l vs Grupo 2: $\mathrm{Chi}^{2}=7,04 \mathrm{p}<0,008$

OR: 1,0 basal

$\begin{array}{rr}1,2 & 3 \text { meses } \\ 2,0 & 6 \text { meses } \\ 2,1 & 9 \text { meses } \\ 3,2 & 12 \text { meses }\end{array}$

Figura 4. Adherencia al Programa de los 3 Grupos.

\section{Discusión}

El objetivo de este estudio fue evaluar retrospectivamente el resultado de una intervención clínica en niños obesos que participaron de un protocolo que abordaba la dimensión psicológica vs la de niños que no participaron de dicho protocolo. Todos los niños fueron similares en sus características de ingreso tanto antropométricas, metabólicas, de hábitos y psicosociales, lo que nos permite compararlos en la respuesta a la intervención.

Las familias muestran, respecto a la tendencia nacional, una mayor proporción de estructura familiar de tipo nuclear y presencia de ambos padres $^{24}$. Estos perciben normalidad respecto al desarrollo físico, psicológico y social de sus hijos. De la misma forma, no perciben dificultades en la conducta alimentaria en etapas tempranas y la proporción de rechazo alimentario no supera lo descrito en otro estudio nacional ${ }^{25}$. Todo esto da cuenta de un grupo bastante normal con rela- ción al perfil psicosocial descrito para población chilena.

Con relación a características conductuales, los padres distinguen en sus hijos atributos fundamentalmente positivos, reconociendo algunas conductas "negativas" relacionadas con labilidad emocional y dificultades de control. Estas últimas dicen relación con la expresión conductual de patrones "desregulatorios" y constituye un resultado interesante de analizar y considerar. La investigación en obesos describe comportamientos que expresan "desregulación" en los mecanismos de control conductual y regulación emocional, así como patrones de funcionamiento familiar caracterizados por dificultades en el control y expresión emocional de sus miembros ${ }^{18-20}$. Estos antecedentes fundamentan los aspectos específicos que integró y abordó el protocolo de intervención psicológica.

En este estudio, todos los grupos, los intervenidos (G1I), los que rechazaron la intervención (G1NI) y el grupo control, tuvieron respuestas positivas en lo referente a la disminución del peso 
corporal y a la mejoría de sus hábitos de ingesta y AF, aunque estas fueron significativas sólo en la mejoría de los hábitos en el grupo intervenido y en el control. El 60,3\% de los niños invitados a participar en el protocolo de intervención psicológica no asistió a los talleres o asistieron a un número de sesiones considerado insuficiente al corte de intervención establecido; de la misma forma, 79,5\% de los padres de este grupo no asistió o asistió a 1 sola de las 3 sesiones para padres, lo que podría reflejar una escasa valoración del apoyo psicológico, por parte de la familia, en la tarea de cambiar conductas de ingesta y estilos de vida.

El protocolo de intervención psicosocial no parece constituir un especial aporte en la mejoría del peso, pero si en la conducta de modificar el gasto, lo que eventualmente tendrá un impacto en el peso. La falta de diferencia entre los grupos, con relación a las modificaciones del peso corporal, podría deberse a que los resultados presentados corresponden a los niños que completaron el programa. Sin embargo, en el contexto de una intervención integrada, y en la necesidad de identificar el aporte específico de cada disciplina, podemos señalar que la intervención psicosocial constituye un importante aporte en el incremento de la adherencia al tratamiento. Una de las principales barreras para el logro de las metas en los programas de intervención, es la baja adherencia lo que no permite cumplir con los protocolos establecidos. Los dos grupos que no recibieron la intervención psicológica tuvieron una alta deserción a los 6 meses $(84,1 \%$ y $69,7 \%$ respectivamente), similar a la descrita en otros estudios nacionales sin intervención psicológica ${ }^{26}$. En el grupo intervenido psicológicamente, la deserción a los 6 meses fue sólo $37,9 \%$ y $24 \%$ completó los 12 meses del programa. Una de las principales barreras en tratamiento de la obesidad infantil es la baja adherencia, la que se asocia al incremento del peso; de esa forma, la sola permanencia en el programa constituiría un objetivo terapéutico en $\mathrm{si}^{27}$.

La mayor adherencia del grupo intervenido podría ser el resultado de definir a la familia como unidad de intervención y constituye a nuestro parecer el principal aporte de la intervención psicológica en este protocolo clínico; a su vez y en plena concordancia con la especificidad de la intervención, constituye un indicador de regulación y control. Los actuales patrones de alimentación y actividad física constituyen una expresión más de cambios fundamentales en nuestros estilos de vida que incluye además importantes cambios en la organización social; entre otros, el debilitamiento del tejido social ${ }^{28}$. En este escenario la familia, unidad social fundamental, aparece debilitada y con escasos recursos de apoyo para hacer frente a sus tareas más esenciales: el cuidado y protección de sus miembros. Al respecto, los programas que integran el trabajo con niños y padres obtienen mejores resultados ${ }^{29,30}$. Las intervenciones "basadas en la familia" varían desde aquellas focalizadas en fortalecer el manejo de los padres en torno a la alimentación y actividad física, hasta aquellas que apuntan a fortalecer aspectos más amplios del funcionamiento familiar, relacionado con las tareas de la crianza ${ }^{30}$. Nuestra intervención se corresponde con estas últimas. El hacer frente a situaciones de salud especiales, demanda el despliegue de nuevos recursos, tanto como la reorganización del sistema familiar. De esta manera, el equipo de salud debe constituirse en un referente para la construcción de respuestas alternativas en materia de hábitos familiares de vida saludable. Esta tarea debe tener presente tanto el abordaje psicoeducativo como la necesidad de fortalecer las unidades familiares, que presentan mayores dificultades en las funciones de control y regulación de sus miembros.

Una debilidad de este estudio es la potencial influencia de la conformación de los grupos comparados en predeterminar los resultados obtenidos. Si bien, la intervención psicológica es parte del protocolo definido para todos los niños que ingresan al programa, podrían actuar como criterio excluyente el factor económico, al corresponder para cada atención un arancel de costo de los consultantes y la disponibilidad de tiempo para asistir a estas intervenciones. Otros antecedentes que pudiesen actuar como variables de confusión no resultan evidentes, ya que respecto de variables de orden familiar, y de aquellas relativas a características del desarrollo y conducta del niño (a) se aprecia bastante homogeneidad y similitud entre los grupos comparados.

Nuestros resultados confirman la presencia de conductas que manifiestan "desregulación" en los mecanismos de control conductual y emocional del obeso, así como que los tratamientos con enfoque y manejo integral que incluye el apoyo psicológico, aumentan la adherencia. Sin embargo, es necesario evaluar la especificidad en la formulación de intervenciones psicosociales. El 
abordaje de los cambios a nivel individual, familiar y social constituye aún un desafío a delimitar, de manera de elaborar programas de costo beneficio replicables a nivel de la salud pública. Es importante considerar la focalización en grupos de mayor riesgo biológico y la posibilidad de insertar estas intervenciones dentro del sistema escolar, ya que esto podría mejorar la adherencia del niño y la familia.

\section{Referencias}

1. Vio F, Kain J. Epidemiología de la Obesidad en Chile. En: Obesidad un enfoque integral. Eds. Cruchet S., Rozowski J. 2007; pp 20-31.

2. Muzzo S, Cordero J, Ramírez I, Burrows R. Trend in nutritional status and stature among school age children in Chile. Nutrition 2004; 20: 867-973.

3. Kain J, Burrows R, Uauy R. Obesity trends in children and adolescents: Basic Determinants. En: Obesity in Childhood and adolescent. Ch. Chen, W. Dietz. Ed. Nestle Nutrition Worshop Series Pediatric Program 2002; 49: 45-63.

4. Rojas J, Uauy R. Evolución de las normas de alimentación y nutrición del Programa Alimentario y cambios en el estado nutricional de preescolares beneficiario de la JUNJI. Rev Chil Nutr 2006; 33: 91-101.

5. Kain J, Lera L, Rojas J, Uauy R. Obesidad en preescolares de la Región Metropolitana de Chile. Rev Med Chile 2007; 135: 63-70.

6. Olivares S, Kain J, Lera L, Pizarro F, Vio F, Moron C. Nutritional status, food consumption and physical activity among chilean school children. A descriptive study. Eur J Clin Nutr 2004; 58: 1278-85.

7. Vásquez F, Salazar G. Patrón de actividad física en un grupo de preescolares obesos asistentes a jardines infantiles de la JUNJI, evaluados con sensor de movimiento. Rev Chil Nutr 2005; 32: 110-7.

8. Burrows R, Montoya A, Gattas V, Díaz E, Sciaraffia V, Lera L. Hábitos de ingesta y actividad física en escolares de enseñanza básica y media según tipo de establecimiento al que asisten. Rev Med Chile 2008; 136: 53-63.

9. Olivares S, Bustos N, Lera L, Zelada ME. Estado nutricional, consumo de alimentos y actividad física en escolares mujeres de diferentes NSE de Santiago. Rev Med Chile 2007; 135: 71-8.

10. Ferranti SD, GauvreauuK, Ludwig DS, Neufeld EJ, Newburger JW, Rifai N. Prevalence of the metabolic syndrome in american adolescents. Circulation 2004; 110: 2494-7.
11. Jelliffe CJ, Janssen I. Development an Age-Specific adolescent metabolic síndrome. Criteria that are linked to ATP III and IDF criteria. J Am Coll Cardiol 2007; 49: 891-8.

12. Burrows R, Weisstaub G, Ceballos Z, Gattás V, Leiva L, Lera L, et al. Síndrome metabólico en niños y adolescentes: asociación con sensibilidad insulinica, grado y distribución del sobrepeso. Rev Med Chile 2007; 135: 174-81.

13. Prevention of Pediatric Overweight and Obesity. Policy Statement. Committee on Nutrition. American Academy of Pediatrics. Pediatrics 2003; 112: 424-30.

14. Speiser PW, Rudolf MCJ, Anhalt H, Camacho-Hubner C, Chiarelli F, Eliakin A, et al. Consensus Statement: Childhood Obesity. J Clin Endocrinol Metab 2005; 90: 1871-87.

15. Screening and Intervention for overweight in children and adolescent: Recomendation Statement. US Preventive Task Force. Pediatrics 2005; 116: 205-9.

16. Barlow SE, Dietz WH. Obesity evaluation and treatment: Expert Commitee Recomendations. Pediatrics 1998; 102: e.p.29

17. Aymamí N, Serrano F. Personalidad y relaciones interpersonales en la obesidad. En: Trastornos de la Alimentación. Anorexia nerviosa, bulimia y obesidad. Ed. Masson S.A. 1997; pp 242-9.

18. Ganley R. Family Patterns in Obesity: With consideration of emotional eating and restraint. Family Sistems. Medicine 1992; 10: 181-99.

19. Wilkins S, Kendrick O, Stitt K, Stinett N, Hammarlund V. Family functioning is related to overweight in children. J Am Diet Assoc 1998; 98: 572-5.

20. Weststrate J, Deurenberg P. Body composition in children: proposal for a method for calculating body fat percentage from total body density or skinfold-thickness measurements. Am J Clin Nutr 1989; 50: 1104-15.

21. National Health, Lung and blood Institute. Report of de second task force on blood pressuere en children-1987. Pediatrics 1987; 79: 1-2.

22. Godard C, Rodríguez MP, Díaz N, Lera L, Salazar G, Burrows R. Valoración de un Test para evaluar hábitos de actividad física en escolares. Rev Med Chile 2008; 136 : 1155-62.

23. Gungor N, Saad R, Janosky J, Arslanian S. Validation of surrogate estimates of insulin sensitivity and insulin secretion in children and adolescents. J Pediatr 2004; 144: 47-55.

24. Instituto Nacional de Estadísticas (INE). XVII Censo Nacional de Población y VI de Vivienda. Chile, 2002.

25. Salas MI, Pizarro F. Problemas de Rechazo alimentario en Lactantes. Rev Chil Pediatr 1998; 25: 45-50. 
Intervención psicológica en niños obesos - M. I. Salas et al

26. Moraga F, Rebollo MJ, Bohórquez P, Cáceres J, Castillo C. Tratamiento de la obesidad infantil: Factores pronósticos asociados a una respuesta favorable. Rev Chil Pediatr 2003; 74: 374-80.

27. Barja YS, Núñez NE, Velandia AS, Urrejola NP, Hodgson BMI. Adherencia y efectividad a mediano plazo del tratamiento de la obesidad infantil. Rev Chil Pediatr 2005; 76: $151-8$
28. Programa de las Naciones Unidas para el Desarrollo Humano (PNUD). Las paradojas de la Modernización. Informe Desarrollo Humano en Chile, 1998.

29. Epstein L, Myers M, Raynor H, Saelens B. Treatment of pediatric obesity. Pediatrics 1998; 101 (3Pt. 2): 550-70.

30. Nowicka P, Flodmark C. Family in pediatric obesity management: A literatura review. Int J Pediatr Obes 2008; 3: 44-50. 